This item was submitted to Loughborough's Research Repository by the author.

Items in Figshare are protected by copyright, with all rights reserved, unless otherwise indicated.

\title{
A dynamic curation method for manufacturing-related knowledge
}

\section{PLEASE CITE THE PUBLISHED VERSION}

http://dx.doi.org/10.1080/00207543.2016.1222089

\section{PUBLISHER}

(c) Taylor and Francis

\section{VERSION}

AM (Accepted Manuscript)

\section{PUBLISHER STATEMENT}

This work is made available according to the conditions of the Creative Commons Attribution-NonCommercialNoDerivatives 4.0 International (CC BY-NC-ND 4.0) licence. Full details of this licence are available at: https://creativecommons.org/licenses/by-nc-nd/4.0/

\section{LICENCE}

CC BY-NC-ND 4.0

\section{REPOSITORY RECORD}

Wood, Robert. 2016. "A Dynamic Curation Method for Manufacturing-related Knowledge”. Loughborough University. https://hdl.handle.net/2134/23487. 


\section{A DYNAMIC CURATION METHOD FOR MANUFACURING-RELATED KNOWLEDGE}

R L Wood

Wolfson School of Mechanical, Manufacturing and Electrical Engineering, Loughborough University, Loughborough, Leicestershire, UK

R.L.Wood@lboro.ac.uk

ABSTRACT

A method for the dynamic curation of manufacturing-related knowledge is proposed, based on the impact of successive paradigm introduction on the network structure within manufacturing companies. This draws together manufacturing system structure in terms of interacting component network types, the nature and consequences of knowledge silos and the underpinning dichotomous influence of language. The need and opportunities for an objective- rather than subjective paradigm-based view of manufacturing are identified, leading to a curation process in which paradigms and other knowledge specialisms are different viewpoints based on particular models of manufacturing processes and resources. The consequences of this are explored in terms of knowledge silo reduction, improved communication within component social- and information networks, increased operational resilience and better informed decision-making for future business.

\subsection{INTRODUCTION}

Knowledge is a significant proportion of a company's overall wealth, underpinning- and diffusing throughout all of its activities and aspirations. A significant proportion of this knowledge is typically hidden or unknown [1]. According to the MAKE research program [2], companies that proactively recognise and exploit their existing- and new knowledge significantly outperform those that are less conscious of this valuable asset. In comparison to its contemporary society, manufacturing has always been complicated because of the need for disparate specialised knowledge and skills, managed through the division of labour $[3,4]$. As manufacturing companies have grown more diverse, larger and more complicated, the number of specialisms, along with their interactions, has increased, making manufacturing increasingly knowledge intensive and also knowledge diverse. This is exemplified in fig.1 which highlights the approximate chronological introduction of new paradigms since the 1970's. The intensity and diversity of knowledge resulting from this has also caused the growth of significant 'conceptual distance' between different specialists and paradigms. This has often been described in terms of the growth of knowledge silos.

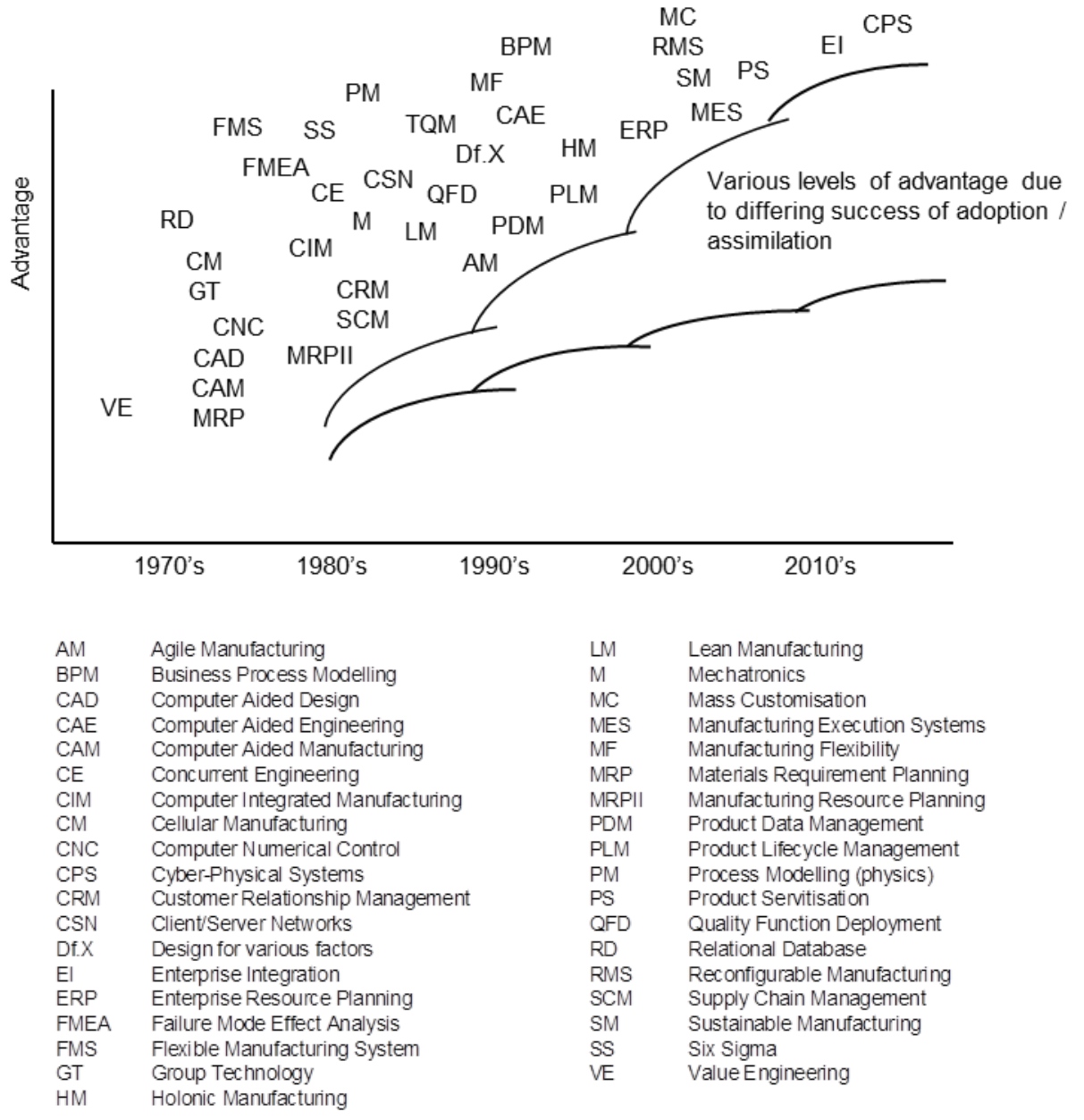

Figure 1. Approximate chronology of first impact of various manufacturing paradigms 
Many of the paradigms in fig. 1 result from the evolution of various thought processes over the history of manufacturing, but only recently enabled by the adoption of various technologies including the microprocessor, client/server networking and relational database software in the 1970's and 1980's. These paradigms offer a range of advantages. Some provide increased automation/assistance, e.g. CAD \& CNC. Others introduce new viewpoints, demands or ways of organising resources, e.g. QFD, Df.X \& FMS. Other paradigms are integrative, subsuming prior viewpoints and ways of organising processes and resources, examples being PDM, PLM, ERP, EI \& CPS. Paradigm introduction is disruptive and it is interesting to note that there is little in the literature to guide the appropriate selection/mix of paradigms for any individual business. While each paradigm aims to achieve advantage through improved capabilities and efficiencies, several unintended consequences arise from their diversity and rate of introduction. Primarily, each paradigm adds a new body of specialised knowledge, new processes and trained/educated practitioners who have specific paradigm-related viewpoints on manufacturing issues. This often leads to difficulty in finding optimum or even good compromise solutions in product development and organisation of manufacturing resources because of conflicting demands and communication difficulties due to lack of shared understanding. Such conflicts are often exacerbated by reliance on computer-based information systems with specialism-specific interfaces that reinforce knowledge silos.

The computer-based technologies supporting paradigm growth have similarly enabled the growth of various Knowledge Management (KM) systems. Reviews of manufacturing-related KM $[5,6]$ suggest that the diversity of KM approaches, together with their foci and objectives is comparable to that found in paradigm development. Numerous KM methods are aligned with particular paradigms and other functional aspects of manufacturing organisations, several methods employing ontological approaches to knowledge definition. Although these methods are locally valuable, an unintended consequence on the wider scale of the whole manufacturing organisation is that they tend to reinforce knowledge silos. This has typically been addressed through other aspects of $\mathrm{KM}$ concerned with interoperability. However, given the range of interacting paradigms and associated specialist viewpoints shown in fig.1, determining unambiguous definitions and translations of knowledge elements is not trivial. This may also be exacerbated by many KM methods only having an implicit recognition of various networks within manufacturing organisations in which knowledge is found and used. As discussed later, the knowledge curation method proposed here complements existing KM methods by offering opportunities to address these issues.

Forecasts such as [7] indicate that the trends shown in fig.1 will continue in some form as manufacturing both influences- and responds to an increasingly complicated and dynamic global environment. In particular, [7] identifies four key categories of future influence: Megatrends; Enablers of future competitiveness; Emerging science and engineering; and Future challenges and opportunities. These influences, along with some of their deeper detail are shown in fig.2, which additionally suggests that manufacturing's global environment will change through manufacturing's influence and through factors and interactions that manufacturing has no control over. Thus, manufacturing will continue to operate as part of an increasingly complicated and dynamic network and the success of individual businesses will be determined by how well they can lever advantage from useful combinations of existing and future paradigms.

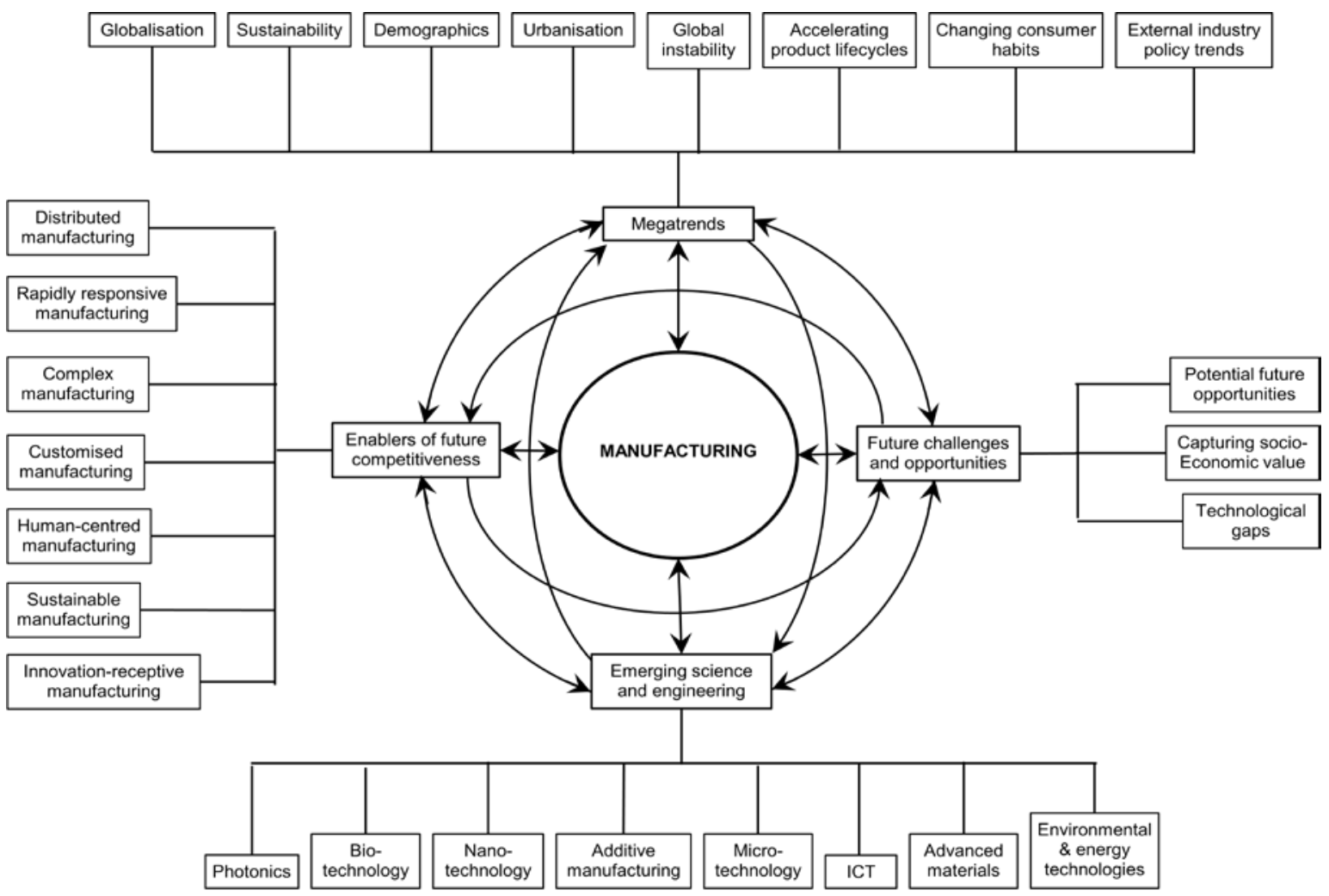

Figure 2. Building on "Emerging trends in global manufacturing industries" [UNIDO 2013] 
To maximise this leverage, it is important to recognise that manufacturing will be increasingly sensitive to knowledge intensity and diversity and that manufacturing-related knowledge needs to be understood objectively, independently of particular paradigms. To develop this argument in more detail, section 2 uses a network approach to explore how paradigm introduction influences organisational structure, functionality and knowledge at different levels of detail. Section 3 explores the relationships between knowledge- and language networks, observing that knowledge silos and the silo mentality are a natural consequence of local communication efficiencies that detract from wider network performance. This section also introduces the notion that any manufacturing process has semantically different identities that depend on the specialism that it is viewed from. Section 4 explores how a carefully designed process for knowledge curation can support significantly improved knowledge sharing and communication between specialisms, offering the possibility of better business performance through improved integration of paradigms.

In what follows, it is useful to discriminate between the following terms:

Knowledge: Including existing direct experiential knowledge involving data, information, skills, theoretical and practical understanding, along with new concepts created in the mind through combinations of experiences and imagination.

Conceptual distance: given several workers, each in a different specialism, how far back in their life history would be found a point of shared education? For example, thermal- and mechanical design are conceptually closer to each other than either is to aesthetic design.

Cognitive distance: the effort or cost to a specialist of learning enough of a different specialism for that new knowledge to be useful. For example, many texts concern metrology and instrumentation but they are unlikely to conveniently package knowledge as it is used in test cells on a production line in a particular company.

Language: Symbols, used according to a set of rules, to reference and communicate knowledge.

Complicated systems: Systems that have predictable behaviour, even though they may be difficult to understand because they may contain many parts with many interactions.

Complex systems: Systems that exhibit unpredictable behaviour and/or produce previously unknown outputs.

\subsection{MANUFACTURING NETWORKS}

This section develops a view of manufacturing organisation based on the presence of several component networks, each involving vertices or nodes representing processes and/or resources, along with links between vertices representing flows or other forms of relationships [8].

\subsection{Component Network types}

Perhaps the most intuitive component network involves flow of material through sequences of transformation processes, as suggested in fig.3(a). In parallel with this, and additionally going beyond it into area such as design, ERP, CRM, etc, there is an information network, fig.3(b), comprising of generally bi-directional information flows and transformation processes. Processes in both of these networks rely on people who form an additional social network, fig.3(c); typically containing clusters aligned with processes in the material and information flow networks. Underpinning these networks, are clusters of knowledge, often referred to as 'silos' forming a fourth network with typically weak or non-existent connectivity between clusters, fig.3(d). Characteristics of such clusters, for example their number and specialism, typically reflect how the business is organised. The consequences of this organisation are helpful, in terms of resource organisation, control and cost monitoring, etc., but also unhelpful in terms of restricting knowledge sharing and capacity for change. In practice, significant interactions occur between these networks, with manufacturing processes typically involving material or information flow as inputs and outputs, along with people using knowledge and interacting with machinery and other tools to generate process outputs, which may include new knowledge generated within the process.

When developing schematics, such as fig.3, care is needed to appreciate the different levels of granularity that can be addressed. Reference architectures [9] and more recent studies, such as [10] [11] [12] [13], serve to highlight the complicated overlapping nature and interaction between the component networks shown in fig.3. An important issue in understanding how to lever advantage from the paradigms shown in fig. 1 is to consider how each influences the structure and dynamic of the component networks in fig. 3 recognising that, for any manufacturing system, its component networks are a consequence of interactions between previously adopted paradigms and organisational decision making. 
(a) Material flow network

People and machines producing components $\&$ products

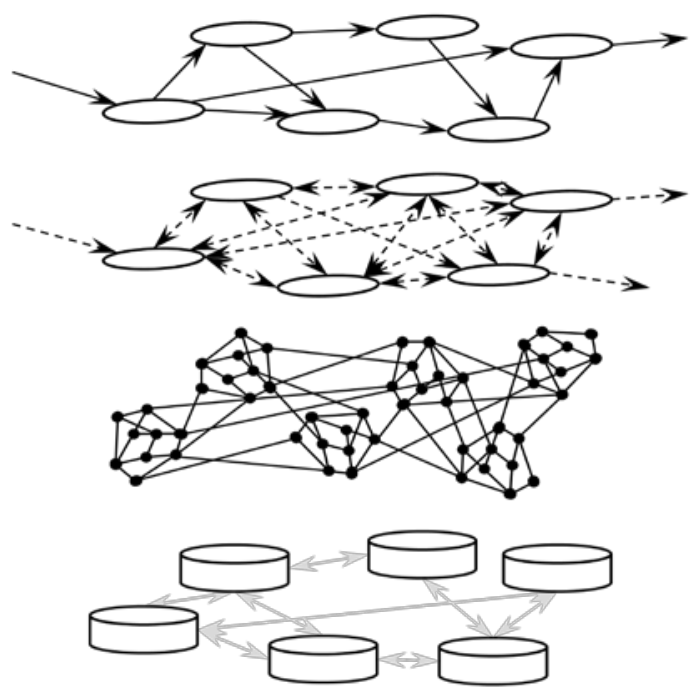

\section{(b) Information network}

Generation and exchange of product

related data; Design, Production

monitoring \& control, CRM, etc.

(c) Social network

Links may be formal or informal \& local or distant between colleagues and friends

(d) Knowledge network

Silos enable nodes in higher level networks. Knowledge may be explicit (Standard

Operating Procedures, User guides, etc.) or

implicit (undocumented methods \& tacit)

Figure 3. Component network types in manufacturing systems

\subsection{Network examples}

To exemplify the nature and interactions between component networks, it is useful to contrast networks associated with ERP and PLM. ERP is typically depicted at the level of detail shown in fig.4, aiming to transform the network of processes shown in fig.4(a) to that in fig.4(b) through the implementation of an ERP information network. This is a relatively coarse granularity and the component nodes are significant networks in their own right.
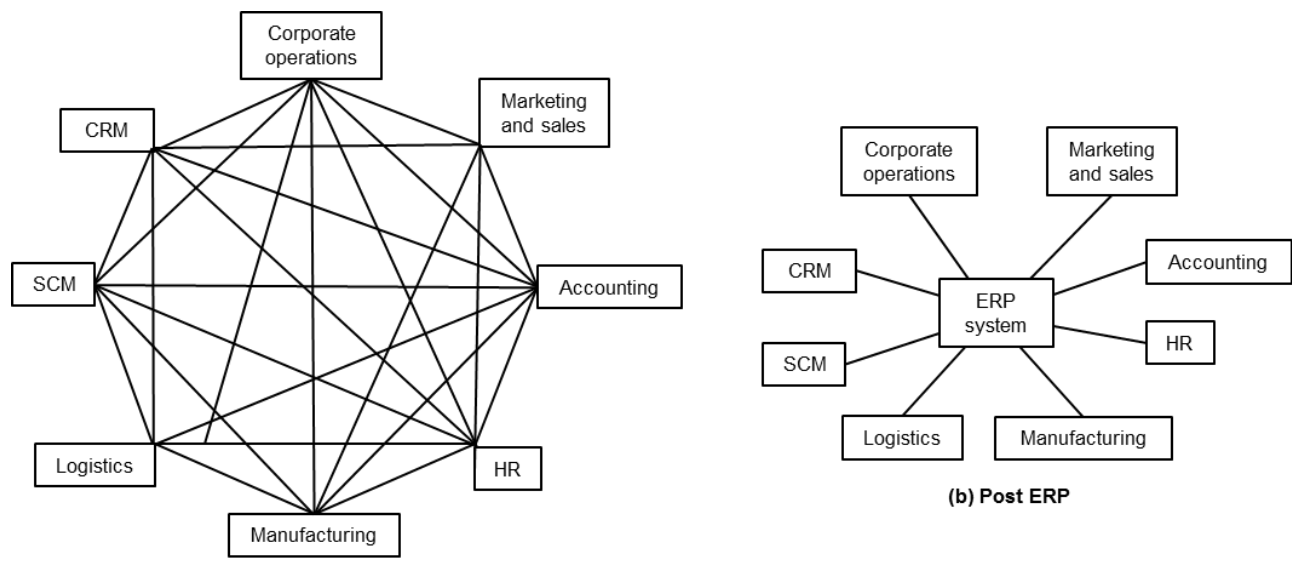

(b) Post ERP

(a) Pre ERP

Figure 4. Restructuring of the information network resulting from ERP introduction

It is well documented that the introduction of ERP systems is disruptive [14], reflected in the simplified connectivity in fig.4(b) over fig.4(a). Key aspects of this simplification are:

- Reduced number of interfaces for each vertex, consistent with fewer network links

- Simplification of processes within each vertex and specialisation of interfaces to the ERP system consistent with the requirements of the new processes

- New opportunities for timely information sharing, providing a more integrated view of operations

The costs of achieving this include:

- New functionality inside the ERP vertex. The functionality of the networked processes within the ERP system is algorithmic, based on defined relationships between possibly diverse but finite well-understood information. Consequently, ERP systems are complicated but should not be complex since they are enabled through the limited use of well-defined language.

- Potential loss of resilience. Network theory indicates that the pre-ERP network, fig.4(a) is a cluster because of a high degree of connectivity between neighbours of any vertex. Clusters have a high degree of resilience to failure or disruption of any vertex or link. In contrast, although the relative simplicity of the post-ERP system, fig.4(b) may appear attractive, 
connectivity between vertices is restricted to flow through the ERP vertex, indicating that no cluster exists and the functionality of the entire network is dependent on a single vertex.

- Reduced connectivity in the parallel social network due to working practices streamlined to comply with the ERP system formalism, further contributing to lower resilience through reduced communication and knowledge sharing.

- Reinforcement of knowledge silos arising from the combined effects of connectivity loss in the information- and social networks, along with specialisation of working practices and interfaces.

- Long term knowledge loss through employee turnover with the eventual loss of an entire 'generation' of staff having any personal experience of the pre-ERP system. This represents a significant loss of intellectual capital that may need to be rediscovered, especially in times of change and reorganisation.

PLM systems are typically depicted, fig.5(a), as a linear or sequential directed network. PLM is an integrative paradigm, establishing complicated relationships between various types of networks. Two important aspects of PLM omitted from fig.5(a) are information flowing upstream from later stages and links to other networks such as ERP. Fig.5(a) also hides numerous other complications, especially in the highlighted design- and planning stages since PLM potentially encompasses many of the paradigms shown in fig.1. This is explored in fig.5(b) which shows around 30 different design specialisms. Interactions and dependencies between these specialisms vary depending on the needs of the product and manufacturing operations, influenced by factors such as product architecture [15], component / sub-system modularity [16], product/service offering [17] and distribution of design effort within the supply chain [16]. The introduction of various design paradigms in recent years has rapidly increased the number of design constraints and dependencies within multi-disciplinary decision making. Creating a design environment which aims to control these issues has led to the development of systems and methods for engineering change management [19] and design conflict resolution [20], effectively steering the design network to the structure shown in fig. $5\{\mathrm{c}$ ). The parallels between this and the ERP enabled network in fig.4(c) are clear and the previous comments concerning the characteristics of this type of network are applicable to design and its management. However, operations management, exemplified here by ERP, and product design are fundamentally different in that the former may be complicated but not expected to be complex, whereas design networks are expected to be complex and are often complicated.

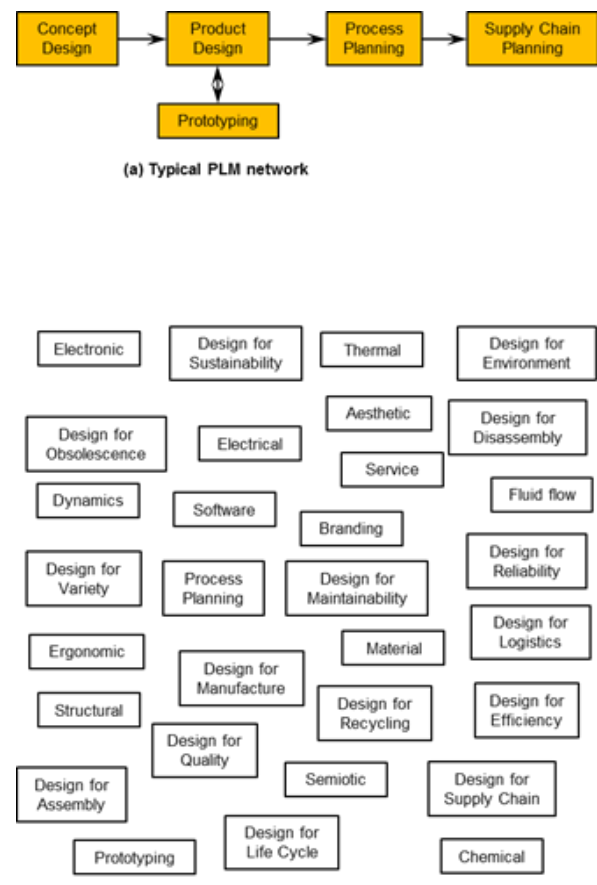

(b) Product design specialisms

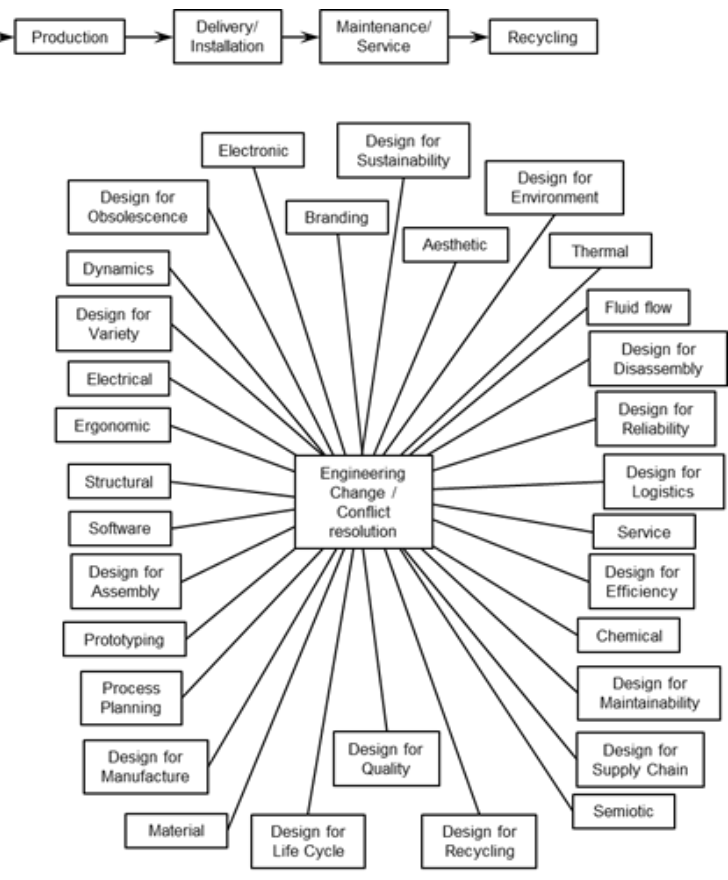

(c) Unclustered Product design network

Figure 5. Expansion of design specialisms within PLM.

Adopting a similar view to [21], design activities can exhibit various forms of complexity and emergent behaviour although the enabling information network may not be intended to be complex. Creative use of knowledge in the synthesis of new design solutions can be considered as 'good' complexity; whereas uncertainty, loss of specialism coordination, not understanding multi-disciplinary consequences of decision-making and the possible need for re-design driven by downstream factors in the product lifecycle can all be considered as 'bad' complexity. Although information networks such as that in fig.5(c) aim to reduce bad complexity, the mechanism needed to achieve this becomes focused at the central vertex concerning change management and conflict resolution. The functionality of the central node depends on an adequate understanding of relevant design disciplines and their dependencies. Having isolated design specialisms from each other, the central vertex also becomes responsible for creative use of knowledge and synthesis of new design solutions. These issues are exacerbated by the restricted viewpoints of designers operating in different specialisms and also by the wide range of possibilities for specialism 
connectivity at different levels of detail. For example, at a higher level, the need for compromise between people focused- and physics based design [22] and, at a lower level, the dependency of component morphology and performance on material properties achievable during production.

Considering the wider consequences of these ERP and PLM examples, it clear that paradigm introduction causes disruption in each of the component networks in fig. 3 and that the offer of improved efficiencies in the material- and information networks can lead to degradation of functionality in the social- and knowledge networks. The consequences of this are various, including increasing difficulty in coordinating resources to achieve targets with growing numbers of influential/relevant paradigms and viewpoints; growing formality of communication reducing opportunities for knowledge sharing as a basis for communication between individuals. Difficulties also arise concerning a company's ability to capture and value its own explicit and tacit knowledge [23], along with its capacity to absorb new knowledge from external sources [1].

\subsection{LANGUAGE, KNOWLEDGE SILOS AND PARADIGM VIEWPOINTS}

In addition to the network types shown in fig.3, language is the venous system that enables their operation and is itself, a complex network [24, 25]. Fundamentally, language enables conceptualisation, communication and use of knowledge within processes and appears in various forms, for example:

- Kanban cards

- Machine tool operator interfaces

- Software user interfaces

- Programming- and mark-up languages

- Standard Operating Procedures (SOPs)

- Documentation of explicit knowledge

- Expression of implicit or tacit knowledge

- Codification of knowledge through ontologies

Languages may appear as rigorous formalisms, as in ontologies [26], programming languages and SOPs; visually through semiotics [25 27] in user- and operator interfaces; and openly expressive in people's thoughts and interactions. The fluidity and expressive power of language enables specification and assimilation of concepts and viewpoints, along with the use of knowledge, information and data for decision making and decision making itself. However, language also plays a major part in restricting manufacturing system performance and development through its use in building specialised bodies of knowledge and reinforcement of knowledge silos in the knowledge network. Specialisms, or silos, arise for two main reasons:

- Through division of labour and organisational design which concentrates resources into the specialist areas needed to enable a company to function. Essentially, this is a particular way of knowing and applying something, for example, a specialised path through a body of knowledge that supports some design or production task.

- Through the introduction of new paradigms, creating additional viewpoints in- and between existing specialisms.

Knowledge silos consist of concepts, internal inter-concept relationships and external relationships with wider aspects of the company. Such knowledge may be stored explicitly in SOPs and published texts, and also tacitly in the experience and knowhow of individuals in the social network. This is both mediated and inhibited by the use and evolution of language. Within any community, including practitioners within a specialism, language evolves towards increasing communication efficiency by maximising meaning whilst minimising communication effort [24]. Acronyms, such as those in fig.1 are a natural consequence of this. However, such refinements tend to inhibit sharing of knowledge between specialist communities due to divergence of specialist languages. This is further exacerbated by the emergence and maturation of different specialist cultures as clusters within the social network that establish the 'world view' of a specialism and particular viewpoints on other aspects of a company and its organisation. These viewpoints are enacted through the information network and impact on the operation of the material network.

Knowledge in any paradigm or specialism concerning manufacturing processes and resources is subjective and can be considered as a particular 'viewpoint'. Such viewpoints involve a mixture of explicit and implicit knowledge and are often hidden in that practitioners may be unaware of their bias and that other viewpoints are possible. Knowledge silos can be considered as the limits of visibility. Building on these points, the general structure of a paradigm can be said to consist of:

- Processes

- Specialised knowledge

- Practitioners

- Viewpoint on existing processes and resources, including those in other paradigms

Many of the paradigms in fig. 1 overlap in that they interact with the same processers and resources within a company. Hence, the viewpoint of each paradigm is an interaction with a sub-set or model of the available knowledge that is relevant to it, along with a narrative of how the paradigm or specialism interacts with the model. Each narrative is expressed using language relevant to the specialism, so that processes and resources have multiple semantic identities. For example, in general terms, processes are: Verbs in Business Process Engineering/Modelling; Nouns in Manufacturing Technology; Alphanumerical values in Finance/Accounting. The knowledge curation approach discussed in the next section exploits many of these points to provide a means of documenting manufacturing related knowledge.

\subsection{DYNAMIC MANUFACTURING KNOWLEDGE CURATION}

The proposed curation method develops the notion that processes and resources have multiple semantic identities, each corresponding to the subjective viewpoint of a specialism. Each viewpoint will see only those attributes of a process or resource that are relevant to it. This suggests that each semantic identity is a model of the process or resource because it only concerns a subset of its attributes. Further to identifying a viewpoint-specific model, it can also be recognised that the 
interaction between the specialism and the process or resource can be documented as a narrative. Thus, any process can be documented by collating the viewpoints that have reason to interact with it to provide benefits including:

- Documentation of the knowledge, practice, etc in all specialisms through their use of processes and resources

- Documentation of processes and resources through their attributes, with narratives identifying all of the contributions that they make within the manufacturing system

- Documentation of processes and resources in a way that is independent of any specialism, yet accommodating the knowledge and needs of all specialisms

- Approaching any process or resource from any specialism viewpoint reveals other specialised viewpoints and their detail

- Models in different viewpoints will typically overlap, with some process or resource attributes being relevant to multiple specialisms

- Depending on the specificity of attribute definitions, processes and resources can be grouped within a taxonomy, e.g. building on previous approaches to process documentation, such as that for production processes in [28]

- Opportunities to inform and augment existing KM methods, especially those concerned with interoperability.

In the spirit of this paper, it is unlikely that a definitive list of process attributes can be produced from a single viewpoint, such as that of the author, so the following are presented as illustrative examples. The starting point is to recognise a number of knowledge bases pertaining to: Processes, Machines, Employees, Materials, Products, Production and Infrastructure. Fig. 6 shows these knowledge bases, each containing a prototype structure for attributes within it.

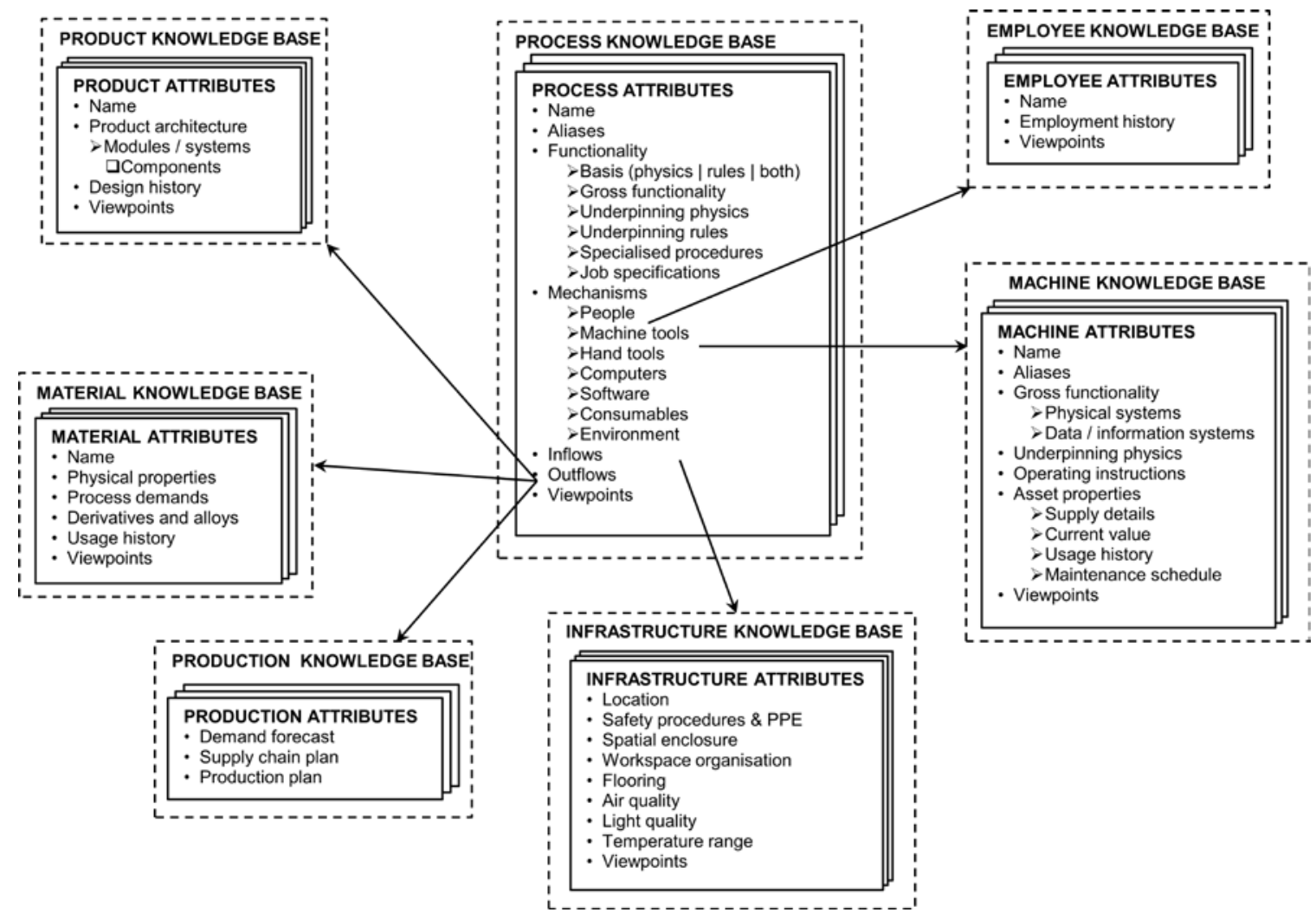

Figure 6. Example knowledge bases at the core of dynamic knowledge curation

The following explores the rationale of the proposed curation method, initially concentrating on those attributes that contribute to models, followed by consideration of various individual viewpoints. Fig. 6 focuses on processes as the core theme, with various attributes linking to elements within other knowledge bases. The first attribute in a knowledge base element is typically "Name", not only identifying the element within a specialism but also representing its nett functionality or identity. "Name" can also be accompanied by one or more aliases, each relevant to a different specialism viewpoint. Each of the remaining process attributes has multiple sub- attributes. Process "Functionality" groups together a number of more detailed attributes that document aspects of the task(s), along with their underpinning principles, that are performed within the process. This includes "basis", indicating that the process is intended to primarily manipulate material (physics), information (rules) or both. Examples of these could be packing of products into boxes, updating of an employee's record in HR, or prototype development and testing, respectively. "Gross functionality" enables documentation of the detailed activities that take place within the process. This typically identifies the process to be a system consisting of more elemental interacting processes that perform constituent tasks. For example, loading and unloading of parts from a machine tool or recognition of the specialisms and their interactions in a multi-disciplinary design team. Constituent processes are also documented individually in the Process Knowledge Base. Underpinning physics and rules document the knowledge and understanding that constitute the process specialism, 'physics' relating to processes that primarily manipulate material and 'rules' to those that manipulate information. These attributes are the fundamental reason for the conceptual distance between the knowledge of practitioners within different specialisms. 
'Specialised procedures' are a distillation of underpinning physics and rules into standard operating procedures, codes of practice or algorithms, along with other relevant explicit and captured tacit knowledge. 'Job specifications', relevant to the HR specialism, are summaries of knowledge and previous experience needed by people to perform their role within the process.

The process "Mechanisms" attribute is concerned with resources needed to perform the required process, exemplified in fig.6 by the division into people, machine- and hand tools, computers and software, consumables and the working environment. These attributes typically link to other knowledge bases. For example "People" identifies particular employees via an Employee knowledge base which itself may link to other HR resources. The "Machine tool" attribute itemises relevant machinery, providing links to the Machine knowledge base to access further detail, as discussed below. "Computer" and "Software" attributes can accommodate many tools depending on process type, ranging from specialised workstations and design software, paradigm-centred tools such as ERP software and utilities for word processing, etc., on desktop- and notebook computers. Similarly, "Consumables" include electricity, gas, water, specialised devices and chemicals, and office supplies. The final mechanism attribute "Environment" suggested in fig. 6 is concerned with those factors needed to ensure a safe and appropriate environment in which the process takes place.

The process "Inflow" and "Outflow" attributes capture both material- and information flows entering and leaving the process. For example, a CNC process has inflows of material and processing instructions and outflows of material and process performance and events. In contrast, a stress analysis process operates solely in the information network with inflows including geometry, material properties and boundary conditions, and outflows such as displacement, strain and stress distributions. When the "Gross functionality" of a process indicates that it consists of several elemental processes, as in the case of a flowline found, for example in the manufacture of automobiles, white goods, semiconductors and confectionary, inflows and outflows can be identified acting at different levels of granularity. Multiple synchronised material and information inflows occur at the whole flowline level, with an incrementally complete product inflowing to successive elemental processes. Process "inflow" and "Outflow" attributes are particularly important in recognising how processes take part in the component network types shown in fig.3. More generally, the overall collection of process attributes link to a range of other knowledge bases that can draw the content of other paradigm-based systems, such as those in fig.1, into the curation process.

In the Machine knowledge base, the "Name" and "Aliases" attributes serve the same purpose as those for processes. The "Gross functionality" and "Underpinning physics" attributes document the machine as an engineered system and in terms of physical principles, respectively. Gross functionality works at different levels of granularity. For example flow lines and flexible manufacturing systems can be decomposed into component networks for material and information flow involving other machines that can be further decomposed into component systems. Such decomposition also accommodates data acquisition systems, control- and other information processing systems within the machine, such as sensors and actuators, along with the processing of instructions in CNC machines. Separation into component networks is achieved through the "Physical systems" and "Data/information systems" attributes, which can also document relevant interface characteristics and behaviour, for example: between sub-systems and components within a machine and between a machine and its external environment.

In contrast, the "Underpinning physics" attribute addresses the physical principles on which the machine depends. This includes processed material response to the influence of the machine, interfaces between the machine and the processed material and the physics underpinning the operation and interactions of machine components and sub-systems. Documented phenomena can include, for example, residual stress development in quenching processes; nucleation and freezing in casting processes, chip formation in machining, etc. Physics underpinning machine sub-systems can include kinematic and dynamic characteristics of robots, energy conversion- and delivery systems in machine tools, lasers and welding torches, etc. The "Underpinning physics" attribute also includes metrology which is fundamental to machine control, including sensing and actuation.

The "Operating instructions" attribute accommodates vendor-supplied documentation, operator knowledge gained from training and previous experience, along with other documented SOPs.

All processes and resources in the knowledge bases in fig.6 have a "Viewpoints" attribute which documents how various specialisms interact with the relevant model through a narrative. Narratives may take different forms, including SOPs, along with material- and information transfer, and interactions between individuals in- and between the various component networks in fig.3. For example, a machining process involving a CNC machine and operator can be viewed from the perspectives of design concerning its ability to achieve required shape; HR in terms of operator skills, experience and salary; ERP in terms of process planning and processing instructions; Maintenance concerning effective machine operation and Apprentice training in terms of attaining competence and understanding.

Paradigm-related viewpoints of a process are typically mediated through the use of other processes. For example, a designer's view of a production process may occur through the application of various Df.X principles, essentially aiming to minimise process use. In contrast, a Lean practitioner would view the same process as a system, aiming to maximise its simplicity

As with several other attributes, "Viewpoints" can also be documented at various levels of granularity. For example, the viewpoint of the Lean paradigm can be contrasted with the viewpoint of an individual Lean practitioner. The former focuses on the Inflow and Outflow attributes of processes in the Lean Paradigm being those 'target' processes that are being made leaner. In contrast, the viewpoint of a Lean practitioner will be more concerned with understanding and changing those attributes within the target processes that offer greatest efficiency achievement. Interactions between the Lean practitioner and people involved with target processes will grow the component social network shown in fig.3, but this is unlikely to be documented in the paradigm level viewpoint. Thus, both model and narrative within a viewpoint depend on granularity.

Viewpoints may also be instigated by the viewed process. For example, HR or Asset Management departments implementing 'front desks' or web site 'front pages' aim to instil a homogeneous viewpoint of themselves when seen from other specialisms. The model in this viewpoint is relatively minimalistic, initially concentrating on Inflow and Outflow attributes and aiming to reveal only those attributes essential to facilitate any interaction. If an initial interaction develops into a more detailed dialogue between individuals, more subjective viewpoints belonging to each individual will develop and add to the component social network. 
Categorisation and grouping of processes and resources provides important support for their understanding and for comparison of viewpoints. The attribute types shown in fig. 6 can accommodate various types of categorisation and grouping based on a range of criteria. This is explored in fig.7 in which an initial structural sub-division of manufacturing leads to taxonomies that capture increasing specialism of processes and materials. Fig.7 also demonstrates process grouping in terms of a part processing route. Additional criteria for process grouping can be the integration of processes into systems such as flow lines, flexible manufacturing systems and other integrative facilities. The processes taxonomy in fig. 7 is based on the process name attribute representing nett functionality. Alternatives to this could be underpinning physics for processes in the material network and underpinning rules for those in the information network, each emphasising possible reuse of knowledge through different viewpoints.

The knowledge curation method discussed here is described as "Dynamic" because it is intended that all employees within a company have roles to play in its development and maintenance since they are the custodians of the knowledge. This may be achieved through various forms of incentives, such as reward structures and ranking within the social network, similar to that in numerous internet-based wikis. The benefits of this are broad, including growth and reinforcement of links in the social network, opportunities for more rapid training and assimilation of new employees, including apprentices, graduates and others developing greater awareness of those who have contributed specialised knowledge and experience; along with various roles for those approaching retirement, possibly through editing and 'gate keeping' of new additions to curated knowledge.

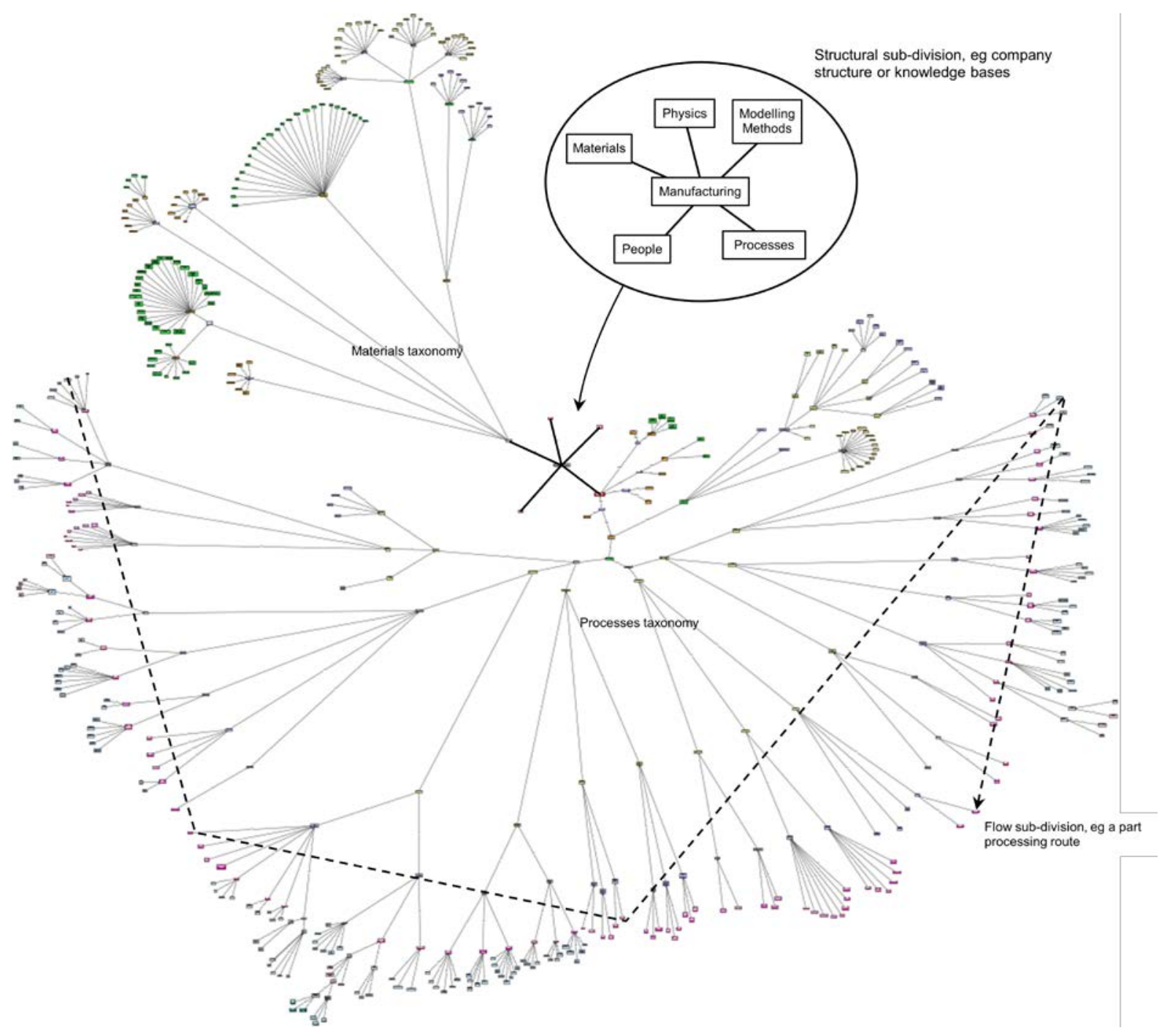

Figure 7. A partial exploratory knowledge map for processes in the component material network

\subsection{DISCUSSION}

The curation method proposed here directly responds to the notion that specialisms and paradigms overlap in their interactions with manufacturing processes and resources, and that the cognitive distance between various specialisms and paradigms can be reduced by drawing their viewpoints together in the form of models and narratives. This provides a new opportunity to reduce conceptual distance between specialists through more effective knowledge sharing, leading to better communication, 
learning, understanding and multi-disciplinary problem solving. This also involves language sharing, further reducing the negative effects of knowledge silos.

These benefits can also be seen in network descriptions of manufacturing, such as the ERP and PLM examples considered in section 2.3. Although these and other paradigms offer benefits in the component material- and information networks, they may also promote loss of resilience and intellectual capital. This occurs, firstly, through removal of previous links between nodes, redefinition of functionality within nodes and increased reliance on paradigm-specific nodes in the information network and secondly, through reduced strength of links in the social network and reinforcement of knowledge silos in the component knowledge network. The knowledge curation method discussed here offers significant scope to offset these effects. This can occur by documenting and drawing together various viewpoints within the knowledge bases shown in fig.6, re-establishing and reinforcing links in the component social network and reducing silo effects in the knowledge network. Perhaps most importantly, this offers the opportunity to reinstate the resilience of pre-paradigm networks through increased connectivity and clustering in the social- and knowledge networks whilst maintaining the benefits that paradigms can bring to the material- and information networks. Interactions between the design specialisms in fig. 5 provide a particularly important example of this, with knowledge curation additionally offering the possibility of new mechanisms for change management and conflict resolution.

More generally for network models, knowledge curation involving elemental process and resource attributes, accommodating multiple viewpoints and different levels of granularity, provides knowledge documentation that cuts across all of the component networks shown in fig.3. Factors that enable this include: Multiple viewpoints occurring in- and between each component network; every viewpoint involving at least one network link and each network link occurring in at least one viewpoint; multiple links between component networks. These factors allow curated knowledge to be integrated with network modelling methods, enabling their specialisation to the detailed modelling of particular company structure and dynamics. This is further supported by several of the knowledge bases shown in fig. 6 providing two-way support for manufacturing. Firstly, providing access to present and historical manufacturing information to support process and resource knowledge curation and, secondly, to enable curated knowledge to support current manufacturing activities. These are significant contributions to the development of Virtual Factory systems [29].

The knowledge curation method proposed here complements and supports existing KM methods by offering opportunities to either incorporate multiple viewpoints into existing methods or to possibly cast existing methods as particular viewpoints within the broader context discussed here. In particular, the proposed curation method may have significant value in enabling a more inclusive approach to reducing knowledge silo effects and resolving interoperability issues within KM.

Considering current paradigms and future trends in manufacturing, the proposed curation method provides a novel approach to recognising and documenting what is known within a company and to enable this knowledge to be used more effectively. This provides a better informed basis for steering the future of the company through more informed decision making and a more cohesive workforce based on better shared understanding. This is important in preparing for a future characterised in fig. 2 by increasingly complicated interactions between various types of environments in which manufacturers will operate, along with the structural- and dynamic changes needed within companies to respond to these demands. It is not clear how the advantage curves in fig.1 will develop, possibly exhibiting diminishing returns on investment in new paradigms with increasing knowledge diversity. Those companies that are more conscious of their knowledge and more effective in exploiting it will be more likely to engineer a better mix of paradigms suited to their corporate aspirations and a future trajectory that follows the upper edge of the advantage spectrum in fig.1.

Recognising that the work reported here is largely conceptual, a number of further steps are currently being explored to demonstrate its benefits and to identify key factors likely to influence its practical implementation. These include the investigation of:

- How knowledge sharing via component networks influences the solution of multi-disciplinary manufacturing problems, exemplified by models of multi-disciplinary design [30, 31].

- Pragmatic implementations of the curation method and identification of the need for underpinning ontologies

- Alternative methodologies for knowledge acquisition and access

- Documenting manufacturing facilities in the Author's department to provide a new teaching resource for Design- and Manufacturing Engineering students

\subsection{CONCLUSIONS}

This paper has developed a view of knowledge curation leading the reader from a high level company summary through subjective paradigms to elemental knowledge possessed by individual workers operating in various component networks. Wide ranging benefits of knowledge curation have been highlighted, including its potential for better understanding of current company structure and operations, improved communication and understanding within the workforce, adoption of new paradigms, planning for the future and, more generally, new opportunities for knowledge-rich modelling of manufacturing systems and processes.

\section{REFERENCES}

1 E Bascavusoglu-Moreau, Q C Li, "Knowledge spillovers and sources of knowledge in the manufacturing sector: literature review and empirical evidence for the UK", Future of Manufacturing Project: Evidence Paper 18, Foresight, Government Office for Science, October 2013 
4 L. Borghans, B. terWeel, "The Division of Labour, Worker Organisation, and Technological Change", IZA Discussion Paper No. 1709, August 2005

5 T. J. Barnett, et al, "Design and development of a classification system for knowledge management tools and methods", Proc. Institution of Mechanical Engineers, Part B: Journal of Engineering Manufacture, 224 (B6), pp. 981-993.

6 D. Baxter, et al, "Managing knowledge within the manufacturing enterprise: An overview", Int. J. Manufacturing Technology Management, Vol18, No. 2, 2009, pp. 183-209.

75 UNIDO, "Emerging trends in global manufacturing industries", United Nations Industrial Development Organisation, Vienna, 2013

86 M. E. J. Newman, "Networks an Introduction”, Oxford University Press, 2015

97 T. J. Williams, PERA and GERAM - Enterprise reference Architectures in Enterprise Integration", Information Infrastructure Systems for Manufacturing II, John Mills and Fumihiko Kimura, eds. 1998 IFIP, Published by Kluwer Academic Publishers

108 A granularity model for balancing the structural complexity of manufacturing systems equipment and layout", Journal of Manufacturing Systems 36 (2015) 7-19

119 S.J. Chuu, "Evaluating the flexibility in a manufacturing system using fuzzy multi-attribute group decision-making with multi-granularity linguistic information", Int J Adv Manuf Technol (2007) 32: 409-421

1210 L Orman, "Granularity as a determinant of system architecture", Systems Practice, Vol. 4, No. 3, 1991

1311 R. Frei et al, "A Complexity Theory Approach to Evolvable Production Systems", Int Conf Informatics and control, automation and robotics, Angers, France, 2007

1412 M. Kennerley, A.Neely, "Enterprise Resource Planning: analysing the impact", Integrated Manufacturing Systems, 12/2 (2001) 103-113

1513 K. M. M. Holtta, "Incorporating design effort complexity measures in product architectural design and assessment ", Design Studies 26 (2005) 463-485

1614 S. N. Joergensen et al, "Reconfigurable Manufacturing Systems as an Application of Mass Customisation", Int. J. Industrial Engineering and Management (IJIEM), Vol.1 No 3, 2010, pp. 111 - 119

1715 G. Vasantha, et al, "A manufacturing framework for capability-based product-service systems design", Journal of Remanufacturing 2013, 3:8

1816 K. J. Peterson et al, "Supplier integration into new product development: coordinating product, process and supply chain design", Journal of Operations Management 23 (2005) 371-388

1917 J. Tavcar, J Duhovnik, "Engineering Change Management in Distributed Environment with PDM/PLM Support", Manufacturing the Future, Concepts - Technologies - Visions, ISBN 3-86611-198-3, pp. 908, ARS/pIV, Germany, July 2006, Edited by: Kordic, V.; Lazinica, A. \& Merdan, M.

$2018 \mathrm{Y}$ Jin, "Argumentation-based negotiation for collaborative engineering design", Int. J. Collaborative Engineering, Vol. 1, Nos. 1/2, 2009

2119 C. Earl et al, "Design Change and Complexity", 2nd Workshop on Complexity in Design and Engineering, GIST Technical Report G2005-1, Department of Computing Science, University of Glasgow, Scotland.

2220 K. Kim, K. Lee, "Industrial designers and engineering designers; causes of conflicts, resolving strategies, and perceived image of each other", Design Research Society Conference, Umeå, Sweden, 2014

2321 T. Haldin-Herrgard, "Difficulties in diffusion of tacit knowledge in organizations", Journal of Intellectual Capital, Vol. 1 Iss 4, 2000 , pp. $357-365$

2422 R. V. Sole et al, "Language Networks: Their Structure, Function, and Evolution", Complexity, Vol 15, No. 6, 2010

2523 D J. B. Plotkin, M. A. Nowak, "Language Evolution and Information Theory", J. theor. Biol. (2000) 205, 147-159

2624 M. Obitko, P. Vrba, V. Marik, "Applications of Semantics in Agent-Based Manufacturing Systems", Informatica 34 (2010) 315-330

2725 S. Hall, "This means this; This means that - A user's guide to semiotics", Laurence King Publishing, 2014

2826 L. Alting, “Manufacturing Engineering Processes”, Marcel Dekker, Inc., 1994

2927 T. Tolio et al,, "Virtual Factory: an Integrated Framework for Manufacturing Systems Design and Analysis", Forty Sixth CIRP Conference on Manufacturing Systems 2013, Procedia CIRP 7 ( 2013 ) 25 - 30 

Science+Business Media, LLC, ISBN 978-0-387-33254-3

\section{FIGURE CAPTIONS}

Figure 1. Approximate chronology of first impact of various manufacturing paradigms.

Figure 2. Building on "Emerging trends in global manufacturing industries" [UNIDO 2013]

Figure 3. Component network types in manufacturing systems.

Figure 4. Restructuring of the information network resulting from ERP introduction.

Figure 5. Expansion of design specialisms within PLM.

Figure 6. Example knowledge bases at the core of dynamic knowledge curation.

Figure 7. A partial exploratory knowledge map for processes in the component material network. 